\title{
Two New Taxoids from the Leaves and Stems of Taxus chinensis
}

\author{
Fu-Sheng WANG* and Gui-Rong SHI \\ College of Pharmacy, Dali University; No. 17, Wanghua Road, Dali 671000, P. R. China. \\ Received April 15, 2009; accepted May 25, 2009; published online June 15, 2009
}

Two new taxoids called 7,13-dideacetyl-9,10-debenzoyl-7 $\beta, 9 \alpha$-p-hydroxylbenzylidenedioxy-taxchinin $C$ (1) and 7,13-dideacetyl-2,9,10-debenzoyl-2-tigloyl-7 $\beta, 9 \alpha$-p-hydroxylbenzylidenedioxy-taxchinin $C$ (2) were isolated from the leaves and stems of Taxus chinensis. They represent the first examples of taxoids containing the 5/7/6membered ring system with $7 \beta, 9 \alpha$-p-hydroxylbenzylidenedioxy groups, the structures of which were elucidated based on spectroscopic analyses, especially 1D and 2D NMR spectra.

Key words Taxus chinensis; taxoid; $7 \beta, 9 \alpha$-p-hydroxylbenzylidenedioxy-taxchinin C

The extensive utilization of paclitaxel as an anticancer agent has stimulated interest in the analysis of the various Taxus species to find alternative sources of paclitaxel or related compounds with improved activity. As a result, natural taxoids are increasingly being isolated and identified. ${ }^{1,2)}$ Taxus chinensis (PIlger) ReHd, indigenous to China, is considered a promising source of taxoids, ${ }^{3-8)}$ and we have investigated the chemical constituents of $T$. chinensis in previous studies. $^{9-12)}$

In our continuing search for new, bioactive natural taxoids, we have reinvestigated the polar neutral fractions of the extracts from the leaves and stems of $T$. chinensis and obtained two new taxoids, 7,13-dideacetyl-9,10-debenzoyl-7 $\beta$, $9 \alpha$-p-hydroxylbenzylidenedioxy- taxchinin $\mathrm{C}(\mathbf{1})$ and 7,13dideacetyl-2,9,10-debenzoyl-2-tigloyl-7 $\beta, 9 \alpha$ - $p$-hydroxyl benzylidenedioxy-taxchinin C (2). Structurally, these two new compounds are the first examples of natural taxoids containing the 5/7/6-membered ring system with $7 \beta, 9 \alpha-p$ hydroxylbenzylidenedioxy groups, although a natural 4,20 $p$-hydroxyl-benzylidenedioxy taxane diterpene and several synthetic analogues have been reported. ${ }^{12-14)}$ In this paper, we report the isolation and structural elucidation of the two new taxoids.

\section{Results and Discussion}

Compound $\mathbf{1}$ was isolated as a white amorphous powder. Its molecular formula was determined to be $\mathrm{C}_{36} \mathrm{H}_{42} \mathrm{O}_{11}$ based on the positive HR-electrospray ionization (ESI)-MS $(\mathrm{m} / \mathrm{z}$ $673.2644[\mathrm{M}+\mathrm{Na}]^{+}$, Calcd 673.2624). The ${ }^{1} \mathrm{H}-\mathrm{NMR}$ spectrum of $\mathbf{1}$ showed four characteristic methyl signals of a taxoid skeleton at $\delta_{\mathrm{H}} 1.05,1.06,1.94$, and 1.95 , and the ${ }^{13} \mathrm{C}-$ NMR spectrum displayed characteristic carbon resonances at $\delta_{\mathrm{C}} 68.4(\mathrm{~s}, \mathrm{C}-1), 45.1(\mathrm{~d}, \mathrm{C}-3), 80.3$ (s, C-4), 38.4 (s, C-8), and 76.0 (s, C-15). From the above-mentioned evidence, it can be deduced that 1 is a taxoid with a 5/7/6-membered ring
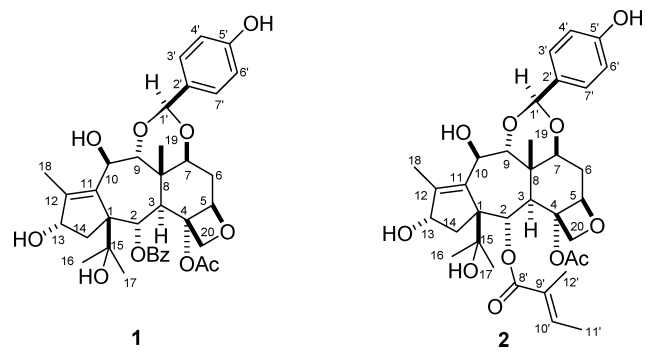

system. ${ }^{3)}$ Moreover, the carbon resonances at $\delta_{\mathrm{C}} 80.3$ (s, C-4) and $75.1(\mathrm{t}, \mathrm{C}-20)$, along with proton signals at $\delta_{\mathrm{H}} 4.02(\mathrm{~d}$, $J=7.5 \mathrm{~Hz}, \mathrm{H}-20 \alpha)$ and $4.42(\mathrm{~d}, J=7.5 \mathrm{~Hz}, \mathrm{H}-20 \beta)$, indicate the presence of an oxetane ring in this molecule. ${ }^{3)}$ Exhaustive comparison of the NMR data (Table 1) of $\mathbf{1}$ with those of the known 7,13-dideacetyl-9,10-debenzoyltaxchinin $\mathrm{C},{ }^{15)}$ showed strong similarities except for the presence of an additional $p$ hydroxylbenzylidenedioxy group in $\mathbf{1}$, based on the carbon resonances at $\delta_{\mathrm{C}} 95.5(\mathrm{~d}), 128.9(\mathrm{~d}, 2 \times \mathrm{C}), 115.4(\mathrm{~d}, 2 \times \mathrm{C})$, $131.5(\mathrm{~s})$, and $158.5(\mathrm{~s})$, together with corresponding proton resonances at $\delta_{\mathrm{H}} 6.03(1 \mathrm{H}, \mathrm{s}), 7.40(2 \mathrm{H}, \mathrm{d}, J=8.5 \mathrm{~Hz})$, and $6.83(2 \mathrm{H}, \mathrm{d}, J=8.5 \mathrm{~Hz})$. The heteronuclear multiple bond connectivity (HMBC) correlations (Fig. 1) of $\mathrm{H}-1^{\prime}$ with $\mathrm{C}-3^{\prime}$ and $\mathrm{C}-7^{\prime}, \mathrm{H}-3^{\prime}$ with $\mathrm{C}-5^{\prime}$ and $\mathrm{C}-1^{\prime}, \mathrm{H}-6^{\prime}$ with $\mathrm{C}-2^{\prime}$, and $\mathrm{H}-7^{\prime}$ with $\mathrm{C}-1^{\prime}$ further confirmed the presence of the $p$-hydroxylbenzylidenedioxy unit. The long-range correlations between $\mathrm{H}-1^{\prime}$ and $\mathrm{C}-7$ and $\mathrm{C}-9$ in the $\mathrm{HMBC}$ spectrum assigned the $p$ hydroxylbenzylidenedioxy group to C-7 and C-9.

The relative stereochemistry of $\mathbf{1}$ was confirmed in a rotating frame Overhauser enhancement spectroscopy (ROESY) experiment, in which cross peaks between $\mathrm{H}-2\left(\delta_{\mathrm{H}} 6.15,1 \mathrm{H}\right.$, $\mathrm{d}, J=7.6 \mathrm{~Hz})$ and $\mathrm{H}-9\left(\delta_{\mathrm{H}} 4.39,1 \mathrm{H}, \mathrm{d}, J=9.6 \mathrm{~Hz}\right)$ and $\mathrm{Me}-19$ $\left(\delta_{\mathrm{H}} 1.95,3 \mathrm{H}, \mathrm{s}\right), \mathrm{H}-9$ and $\mathrm{Me}-17\left(\delta_{\mathrm{H}} 1.05,3 \mathrm{H}, \mathrm{s}\right), \mathrm{H}-13\left(\delta_{\mathrm{H}}\right.$ $4.64,1 \mathrm{H}, \mathrm{dd}, J=11.6 \mathrm{~Hz})$ and $\mathrm{Me}-16\left(\delta_{\mathrm{H}} 1.06,3 \mathrm{H}, \mathrm{s}\right), \mathrm{H}-5$ $\left(\delta_{\mathrm{H}} 4.96,1 \mathrm{H}, \mathrm{d}, J=8.8 \mathrm{~Hz}\right)$, and $\mathrm{H}-6 \mathrm{a}\left(\delta_{\mathrm{H}} 2.4,1 \mathrm{H}, \mathrm{m}\right)$, indicated that $\mathrm{H}-2, \mathrm{H}-9, \mathrm{H}-13$, and $\mathrm{C}-20$ are $\beta$-oriented, while correlations of $\mathrm{H}-3\left(\delta_{\mathrm{H}} 3.06,1 \mathrm{H}, \mathrm{d}, J=7.6 \mathrm{~Hz}\right)$ with $\mathrm{H}-5\left(\delta_{\mathrm{H}}\right.$ $4.96,1 \mathrm{H}, \mathrm{d}, J=8.8 \mathrm{~Hz}), \mathrm{H}-7\left(\delta_{\mathrm{H}} 4.11,1 \mathrm{H}, \mathrm{dd}, J=9.6\right.$, $8.0 \mathrm{~Hz})$, and $\mathrm{H}-10\left(\delta_{\mathrm{H}} 5.4,1 \mathrm{H}, \mathrm{dd}, J=9.6,4.3 \mathrm{~Hz}\right)$ confirmed the $\alpha$-configuration of $\mathrm{H}-3, \mathrm{H}-5, \mathrm{H}-7$, and $\mathrm{H}-10$, respectively (Fig. 1). The ROESY correlations of $\mathrm{H}-1^{\prime}$ with $\mathrm{H}-7$ and $\mathrm{H}-10$ established the $\alpha$-orientation of $\mathrm{H}-\mathrm{1}^{\prime}$. The structure of the
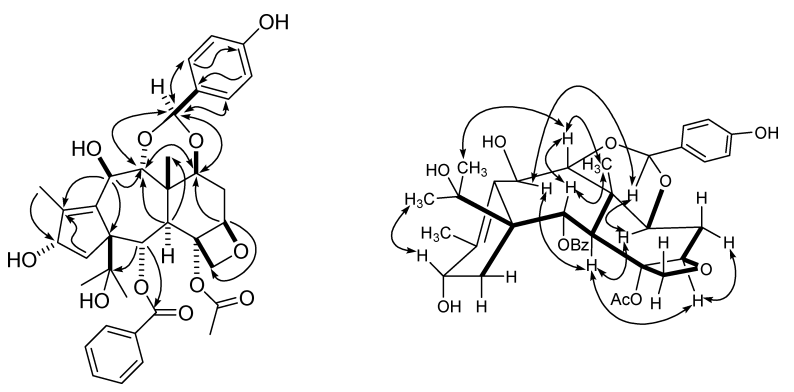

Fig. 1. Key HMBC $(\mathrm{H} \longrightarrow \mathrm{C})$ and ROESY Correlations $(\mathrm{H} \longleftrightarrow \mathrm{H})$ of Compound 1 
Table 1. ${ }^{1} \mathrm{H}$ - and ${ }^{13} \mathrm{C}$-NMR Data of $\mathbf{1}$ and $\mathbf{2}^{a)}$

\begin{tabular}{|c|c|c|c|c|}
\hline \multirow{2}{*}{ No. } & \multicolumn{2}{|l|}{1} & \multicolumn{2}{|l|}{2} \\
\hline & $\delta_{\mathrm{H}}$ mult. $(J=\mathrm{Hz})$ & $\delta_{\mathrm{C}}$ & $\delta_{\mathrm{H}}$ mult. $(J=\mathrm{Hz})$ & $\delta_{\mathrm{C}}$ \\
\hline 1 & - & $68.4 \mathrm{~s}$ & - & $68.4 \mathrm{~s}$ \\
\hline 2 & $6.15(1 \mathrm{H}, \mathrm{d}, 7.6)$ & $70.1 \mathrm{~d}$ & $5.93(1 \mathrm{H}, \mathrm{d}, 7.6)$ & $69.4 \mathrm{~d}$ \\
\hline 3 & $3.06(1 \mathrm{H}, \mathrm{d}, 7.6)$ & $45.1 \mathrm{~d}$ & $2.94(1 \mathrm{H}, \mathrm{d}, 7.6)$ & $45.1 \mathrm{~d}$ \\
\hline 4 & - & $80.3 \mathrm{~s}$ & - & $80.3 \mathrm{~s}$ \\
\hline 5 & $4.96(1 \mathrm{H}, \mathrm{d}, 8.8)$ & $85.3 \mathrm{~d}$ & $4.96(1 \mathrm{H}, \mathrm{d}, 8.8)$ & $85.3 \mathrm{~d}$ \\
\hline $6 \alpha$ & $2.40(1 \mathrm{H}, \mathrm{m})$ & $34.9 \mathrm{t}$ & $1.74(1 \mathrm{H}, \mathrm{m})$ & $34.9 \mathrm{t}$ \\
\hline $6 \beta$ & $1.77(1 \mathrm{H}, \mathrm{m})$ & & $2.37(1 \mathrm{H}, \mathrm{m})$ & \\
\hline 7 & $4.11(1 \mathrm{H}, \mathrm{dd}, 9.6,8.0)$ & $76.4 \mathrm{~d}$ & $4.06(1 \mathrm{H}, \mathrm{dd}, 9.8,7.9)$ & $76.4 \mathrm{~d}$ \\
\hline 8 & - & $38.4 \mathrm{~s}$ & - & $38.4 \mathrm{~s}$ \\
\hline 9 & $4.39(1 \mathrm{H}, \mathrm{d}, 9.6)$ & $84.4 \mathrm{~d}$ & $4.31(1 \mathrm{H}, \mathrm{d}, 9.6)$ & $84.4 \mathrm{~d}$ \\
\hline 10 & $5.40(1 \mathrm{H}, \mathrm{dd}, 9.6,4.3)$ & $63.4 \mathrm{~d}$ & $5.33(1 \mathrm{H}, \mathrm{dd}, 9.6,5.3)$ & $63.4 \mathrm{~d}$ \\
\hline 11 & - & $137.2 \mathrm{~s}$ & - & $137.4 \mathrm{~s}$ \\
\hline 12 & - & $148.3 \mathrm{~s}$ & - & $148.1 \mathrm{~s}$ \\
\hline 13 & $4.64(1 \mathrm{H}, \mathrm{dd}, 6.6,11.6)$ & $77.2 \mathrm{~d}$ & $4.58(1 \mathrm{H}, \mathrm{d}, 3.8)$ & $77.2 \mathrm{~d}$ \\
\hline $14 \alpha$ & $1.99(1 \mathrm{H}, \mathrm{dd}, 14.2,7.3)$ & $40.1 \mathrm{t}$ & $1.85(1 \mathrm{H}$, overlap $)$ & $40.2 \mathrm{t}$ \\
\hline $14 \beta$ & $2.23(1 \mathrm{H}, \mathrm{dd}, 14.2,7.3)$ & & $2.11(1 \mathrm{H}, \mathrm{m})$ & \\
\hline 15 & - & $76.0 \mathrm{~s}$ & - & $76.0 \mathrm{~s}$ \\
\hline 16 & $1.06(3 \mathrm{H}, \mathrm{s})$ & $28.2 \mathrm{q}$ & $1.03(3 \mathrm{H}, \mathrm{s})$ & $25.0 \mathrm{q}$ \\
\hline 17 & $1.05(3 \mathrm{H}, \mathrm{s})$ & $25.0 \mathrm{q}$ & $1.02(3 \mathrm{H}, \mathrm{s})$ & $28.2 \mathrm{q}$ \\
\hline 18 & $1.94(3 \mathrm{H}, \mathrm{s})$ & $11.5 \mathrm{q}$ & $1.90(3 \mathrm{H}, \mathrm{s})$ & $11.5 \mathrm{q}$ \\
\hline 19 & $1.95(3 \mathrm{H}, \mathrm{s})$ & $14.2 \mathrm{q}$ & $1.83(3 \mathrm{H}, \mathrm{s})$ & $12.0 \mathrm{q}$ \\
\hline $20 \alpha$ & $4.02(1 \mathrm{H}, \mathrm{d}, 7.5)$ & $75.1 \mathrm{t}$ & $4.17(1 \mathrm{H}, \mathrm{d}, 7.6)$ & $75.2 \mathrm{t}$ \\
\hline $20 \beta$ & $4.42(1 \mathrm{H}, \mathrm{d}, 7.5)$ & & $4.35(1 \mathrm{H}, \mathrm{d}, 7.6)$ & \\
\hline $\mathrm{OBz}$ & - & $166.9 \mathrm{~s}$ & & \\
\hline$i$ & - & $129.2 \mathrm{~s}$ & & \\
\hline$o$ & $8.08(2 \mathrm{H}, \mathrm{d}, 7.4)$ & $130.3 \mathrm{~d}$ & & \\
\hline$m$ & $7.56(2 \mathrm{H}, \mathrm{t}, 7.5)$ & $129.6 \mathrm{~d}$ & & \\
\hline$p$ & $7.67(1 \mathrm{H}, \mathrm{t}, 7.5)$ & $134.2 \mathrm{~d}$ & & \\
\hline OAc & - & $170.4 \mathrm{~s}$ & - & $170.3 \mathrm{~s}$ \\
\hline $\mathrm{OAc}$ & $2.21(3 \mathrm{H}, \mathrm{s})$ & $22.1 \mathrm{q}$ & $2.13(3 \mathrm{H}, \mathrm{s})$ & $22.0 \mathrm{q}$ \\
\hline $1^{\prime}$ & $6.03(1 \mathrm{H}, \mathrm{s})$ & $95.5 \mathrm{~d}$ & $5.99(1 \mathrm{H}, \mathrm{s})$ & $95.5 \mathrm{~d}$ \\
\hline $2^{\prime}$ & - & $131.5 \mathrm{~s}$ & - & $131.6 \mathrm{~s}$ \\
\hline $3^{\prime}, 7^{\prime}$ & $7.40(2 \mathrm{H}, \mathrm{d}, 8.5)$ & $128.9 \mathrm{~d}$ & $7.37(2 \mathrm{H}, \mathrm{d}, 8.5)$ & $128.9 \mathrm{~d}$ \\
\hline $4^{\prime}, 6^{\prime}$ & $6.83(2 \mathrm{H}, \mathrm{d}, 8.5)$ & $115.4 \mathrm{~d}$ & $6.81(2 \mathrm{H}, \mathrm{d}, 8.5)$ & $115.4 \mathrm{~d}$ \\
\hline $5^{\prime}$ & - & $158.5 \mathrm{~s}$ & - & $158.6 \mathrm{~s}$ \\
\hline $8^{\prime}$ & & & & $168.1 \mathrm{~s}$ \\
\hline $9^{\prime}$ & & & & $129.9 \mathrm{~s}$ \\
\hline $10^{\prime}$ & & & $6.91(1 \mathrm{H}, \mathrm{dd}, 7.1,1.3)$ & $138.9 \mathrm{~d}$ \\
\hline $11^{\prime}$ & & & $1.82(3 \mathrm{H}, \mathrm{s})$ & $14.4 \mathrm{q}$ \\
\hline $12^{\prime}$ & & & $1.88(3 \mathrm{H}, \mathrm{s})$ & $14.1 \mathrm{q}$ \\
\hline
\end{tabular}

a) Recorded in acetone- $d_{6}$ at $400 \mathrm{MHz}$ for ${ }^{1} \mathrm{H}, 100 \mathrm{Mz}$ for ${ }^{13} \mathrm{C}, \delta$ in ppm, $J$ in $\mathrm{Hz}$.

new compound is thus established as a derivative of 7,13dideacetyl-9,10-didebenzoyltaxchinin $C^{3,15}$ ) Therefore, the structure of 1 was identified as 7,13-dideacetyl-9,10-debenzoyl-7 $\beta, 9 \alpha$ - $p$-hydroxylbenzylidenedioxytaxchinin $\mathrm{C}$.

Compound 2, a colorless oil, was assigned the molecular formula $\mathrm{C}_{34} \mathrm{H}_{44} \mathrm{O}_{11}$ as deduced from the quasimolecular ion peak at $m / z$ 651.2780 $[\mathrm{M}+\mathrm{Na}]^{+}$in the HR-ESI-MS (positive), together with the analyses of the ${ }^{13} \mathrm{C}$-NMR and distortionless enhancement by polarization transfer (DEPT) spectra. The NMR data (Table 1) of 2 revealed that it was also a taxane diterpene with a 5/7/6-membered ring system and an oxetane ring, ${ }^{3)}$ featuring four methyl signals of the taxoid skeleton at $\delta_{\mathrm{H}} 1.03,1.02,1.83$, and 1.90. Compound 2 had ${ }^{1} \mathrm{H}$ - and ${ }^{13} \mathrm{C}-\mathrm{NMR}$ spectral patterns similar to those of $\mathbf{1}$. The only difference was the presence of a tiglyol group instead of the benzoyl in 1 due to the carbon resonances at $\delta_{\mathrm{C}} 168.1(\mathrm{~s}$, C-8'), 129.9 (s, C-9'), 138.9 (d, C-10'), 14.4 (q, C-11'), and $14.1\left(\mathrm{q}, \mathrm{C}-12^{\prime}\right)$, together with proton signals at $\delta_{\mathrm{H}} 6.91(\mathrm{dd}$, $\left.J=7.1,1.3 \mathrm{~Hz}, \mathrm{H}-10^{\prime}\right), 1.82\left(\mathrm{~s}, \mathrm{Me}-11^{\prime}\right)$, and 1.88 (s, Me$\left.12^{\prime}\right)$. The tiglyol group was located at $\mathrm{C}-2$ based on the ob-
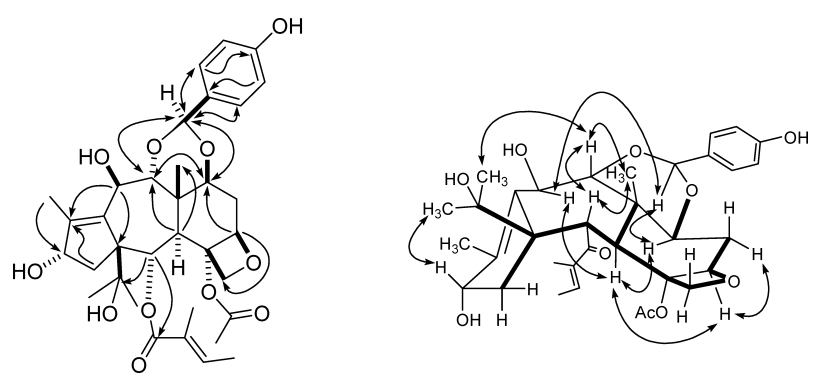

Fig. 2. Key HMBC $(\mathrm{H} \longrightarrow \mathrm{C})$ and ROESY Correlations $(\mathrm{H} \longleftrightarrow \mathrm{H})$ of Compound 2

served cross peak between $\mathrm{H}-2\left(\delta_{\mathrm{H}} 5.93 \mathrm{~d}, J=7.6 \mathrm{~Hz}\right)$ and the ester carbonyl of tiglyol $\left(\delta_{\mathrm{C}} 168.1\right)$ in the HMBC experiment (Fig. 2). The relative configuration of $\mathbf{2}$ was identical to that of $\mathbf{1}$ based on the interpretation of the ROESY spectrum of 2 (Fig. 2). Consequently, the structure of $\mathbf{2}$ to be established as 7,13-dideacetyl-2,9,10-debenzoyl-2-tigloyl-7 $\beta, 9 \alpha$ - $p$-hydroxylbenzylidenedioxytaxchinin $\mathrm{C}$. 


\section{Experimental}

General Experimental Procedures Melting points were determined on an XRC-1 micro melting point apparatus and are uncorrected. Optical rotations were measured with a Horiba SEPA-300 polarimeter. UV spectra were obtained on a UV 2401 PC spectrometer. IR spectra were recorded on a BioRad FTS-135 spectrometer with $\mathrm{KBr}$ pellets. ${ }^{1} \mathrm{H}$ - and ${ }^{13} \mathrm{C}-\mathrm{NMR}$ experiments were performed on a Bruker AM-400 spectrometer $\left({ }^{1} \mathrm{H}, 400 \mathrm{MHz} ;{ }^{13} \mathrm{C}\right.$, $100 \mathrm{MHz}$ ), while 2D NMR spectra were recorded using a Bruker DRX-500 NMR instrument. ESI-MS were taken on a Finnigan-MAT 90 instrument. HR-ESI-MS were measured with a VG Auto Spec 3000 spectrometer. Column chromatography was performed using silica gel (Qingdao Marine Chemical Inc., Qingdao, P.R. China), Lichroprep RP-18 (Merck, Darmstadt, Germany), and Sephadex LH-20 (Pharmacia Fine Chemical Co., Ltd.) HPLC was performed on Hewlett-Packard 1100 Series chromatographs using a Zorbax SB-C18 column $(9.4 \times 250 \mathrm{~mm})$. Fractions were monitored by TLC, and spots were visualized by heating silica gel plates sprayed with $10 \% \mathrm{H}_{2} \mathrm{SO}_{4}$ in $\mathrm{EtOH}$.

Plant Material The leaves and stems of $T$. chinensis (PiLg.) ReHd (Taxaceae) were collected in Liangshang, Sichuan Province, P.R. China, in April 2006 and identified by Prof. Lin Zhongwen. A voucher specimen (No. 200604) has been deposited in the College of Pharmacy, Dali University, P.R. China.

Extraction and Isolation The dried leaves and stems $(20 \mathrm{~kg})$ of $T$. chinensis were extracted three times with $95 \%$ ethanol to give a crude extract $(350 \mathrm{~g})$, after concentration under a vacuum. The residue was chromatographed over a silica gel column eluted with a chloroform-acetone gradient $(9: 1 \rightarrow 1: 1$, and acetone) to give six fractions (A-E, acetone), of which three $(C, D, E)(68 \mathrm{~g})$ were combined and chromatographed on a DM130 column (methanol-water, 9:1) to give a mixture of $56 \mathrm{~g}$. The subfraction was chromatographed over a silica gel column eluted with a chloroform-methanol gradient $(100: 1 \rightarrow 50: 1)$ to afford four subfractions, I-IV Subfraction II was chromatographed on Sephdex LH-20 eluted with $\mathrm{MeOH}$, and a mixture of compounds $\mathbf{1}$ and $\mathbf{2}$ was obtained. The mixture was further chromatographed on a silica gel column eluted with cyclohexane-chloroform-2-propanol $(6.0: 3.5: 0.5 \rightarrow 5.0: 4.5: 0.5)$, and finally purified on Lichroprep RP-18 (methanol-water, $5.5: 4.5$ ) and HPLC (methanol-water, $5.0: 5.0)$ to give compounds $\mathbf{1}(9.1 \mathrm{mg})$ and $\mathbf{2}(4.2 \mathrm{mg})$.

7,13-Dideacetyl-9,10-debenzoyl-7 $\beta, 9 \alpha$ - $p$-hydroxylbenzylidenedioxy-taxchinin $\mathrm{C}(1)$ : White powder; $[\alpha]_{\mathrm{D}}^{20}-18.7^{\circ}\left(c=0.95, \mathrm{CH}_{3} \mathrm{OH}\right)$; UV $\lambda_{\max } \mathrm{nm}$ $(\log \varepsilon)\left(\mathrm{CH}_{3} \mathrm{OH}\right): 202.00$ (4.26); IR $v_{\max } \mathrm{cm}^{-1}(\mathrm{KBr}): 3428,2975,1715$,
$1617,1521,1451,1369,1270,1109,1150,1109,715 ;{ }^{1} \mathrm{H}-$ and ${ }^{13} \mathrm{C}-\mathrm{NMR}$ data, see Table 1; HR-ESI-MS m/z 673.2644 (Calcd for $\mathrm{C}_{36} \mathrm{H}_{42} \mathrm{O}_{11} \mathrm{Na}$, 673.2624).

7,13-Dideacetyl-2,9,10-debenzoyl-2-tigloyl-7 $\beta, 9 \alpha$ - $p$-hydroxylbenzylidenedioxy-taxchinin $\mathrm{C}(2)$ : Colorless oil; $[\alpha]_{\mathrm{D}}^{20}-6.1^{\circ}\left(c=0.10, \mathrm{CH}_{3} \mathrm{OH}\right)$; UV $\lambda_{\max } \mathrm{nm}(\log \varepsilon)\left(\mathrm{CH}_{3} \mathrm{OH}\right): 210.40$ (4.38); IR $v_{\max } \mathrm{cm}^{-1}(\mathrm{KBr}): 3440$, $2973,2928,1707,1630,1520,1369,1254,1168,1104,1070 ;{ }^{1} \mathrm{H}-$ and ${ }^{13} \mathrm{C}-$ NMR data, see Table 1; HR-ESI-MS $m / z 651.2780$ (Calcd for $\mathrm{C}_{34} \mathrm{H}_{44} \mathrm{O}_{11} \mathrm{Na}$, 651.2781).

\section{References}

1) Baloglu E., Kingston D. G. I., J. Nat. Prod., 62 